\title{
S Rehabilitation Reference Center
}

Date of Review: August 18, 2009

\author{
Composite Score: \\ Oviatt Library, Reviewed by: Marcia Henry \\ California State University Northridge \\ 18111 Nordhoff Street \\ Northridge, CA 91330-8327 \\ <mhenry@csun.edu>
}

Christina Mayberry

Oviatt Library, Technical Services

California State University Northridge

18111 Nordhoff Street

Northridge, CA 91330-8328

<christina.mayberry@csun.edu>

\section{Pricing Options}

Rehabilitation Reference Center (RRC) is available exclusively on the EBSCOhost platform. Pricing is determined on an individual basis and depends on the potential number of users, which can vary widely (a small hospital, a multicampus university, a hospital system, or consortia). For academic sites, pricing is based on FTE; for hospitals, the number of beds.

\section{Product Description}

$\mathrm{RRC}$ is a collection of evidence-based information for rehabilitation clinicians: physical therapists, occupational therapists, speech therapists, and audiologists. It is designed to be used at the point-of-care, and allows users to personalize and print patient education and exercise handouts. Clinical reviews are developed by the CINAHL Rehabilitation editorial team, which monitors the content of medical and rehabilitation journals indexed in MEDLINE, CINAHL, other appropriate databases, appropriate Web sites such CDC, and specialty organizations. RRC content and editorial policies are described at Ebsco's Web site $<$ http://www.ebscohost.com/rrc_editorial/default.php $>$.

\section{Critical Evaluation}

\section{CONTENT}

Diseases and Conditions Currently RRC offers 200 clinicallyoriented reviews on rehabilitation topics, but is expected to grow to 600 clinical reviews. RRC incorporates the best available evidence through rigorous systematic surveillance by EBSCO's clinical reviewers. Each clinical review includes indexing/metadata in addition to the title, a range of synonyms, anatomical location/body part affected, ICD codes (9 and 10), reimbursement information when available, presentation signs, and symptoms. Clinical reviews are developed by the CINAHL RRC editorial team and are unique to RRC. The reviews are intended to be used at point of care and are designed so that the therapist can drill down efficiently to the needed information. Figure 2 points out the indexing metadata and contents in a clinical review.

Drug Information Information from AHFS (American Hospital Formulary Service) on over 11,700 drugs and their manufacturers as well as separate monographs for systematic topical and EENT drug formulations are included. Individual drug records include information on usage, dosage and administration, cautions, drug interactions,

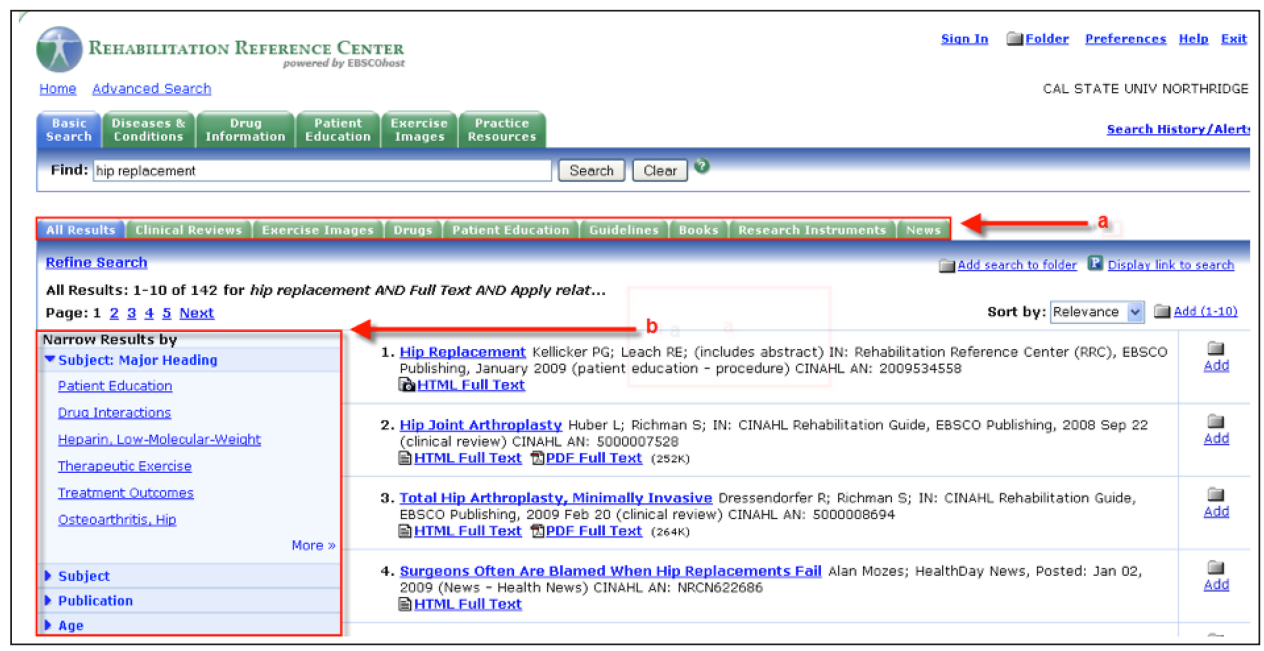

FIGURE 1 RRC retrievals for a search on hip replacement 
FIGURE 2 Example of clinical review with MetaIndexing and Contents

FIGURE 3 Custom Printout options

FIGURE 4 Exercise images retrieved for hip replacement

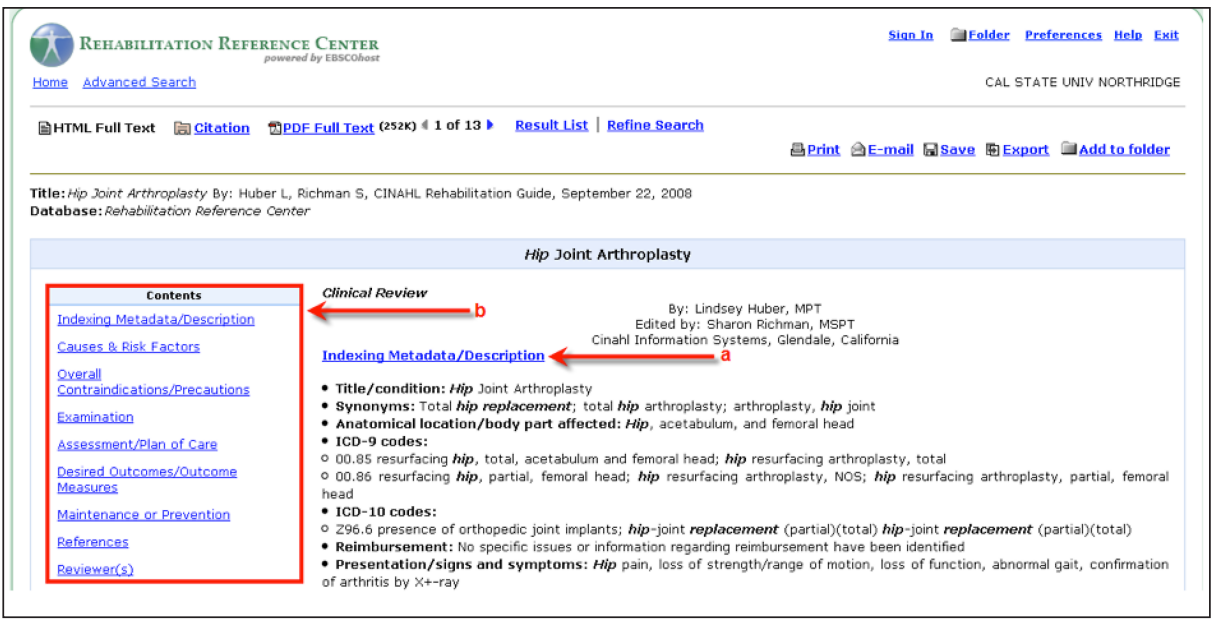
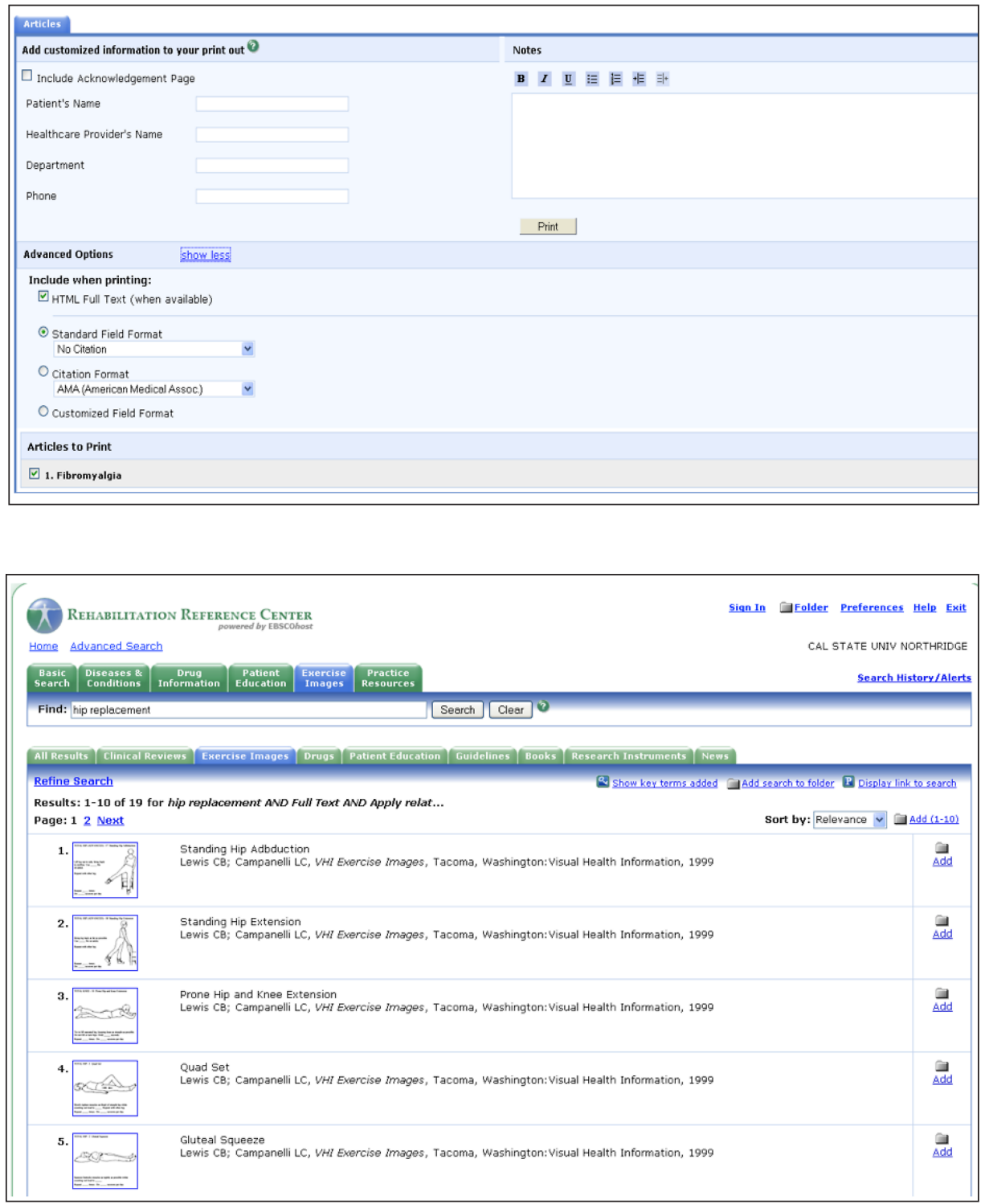


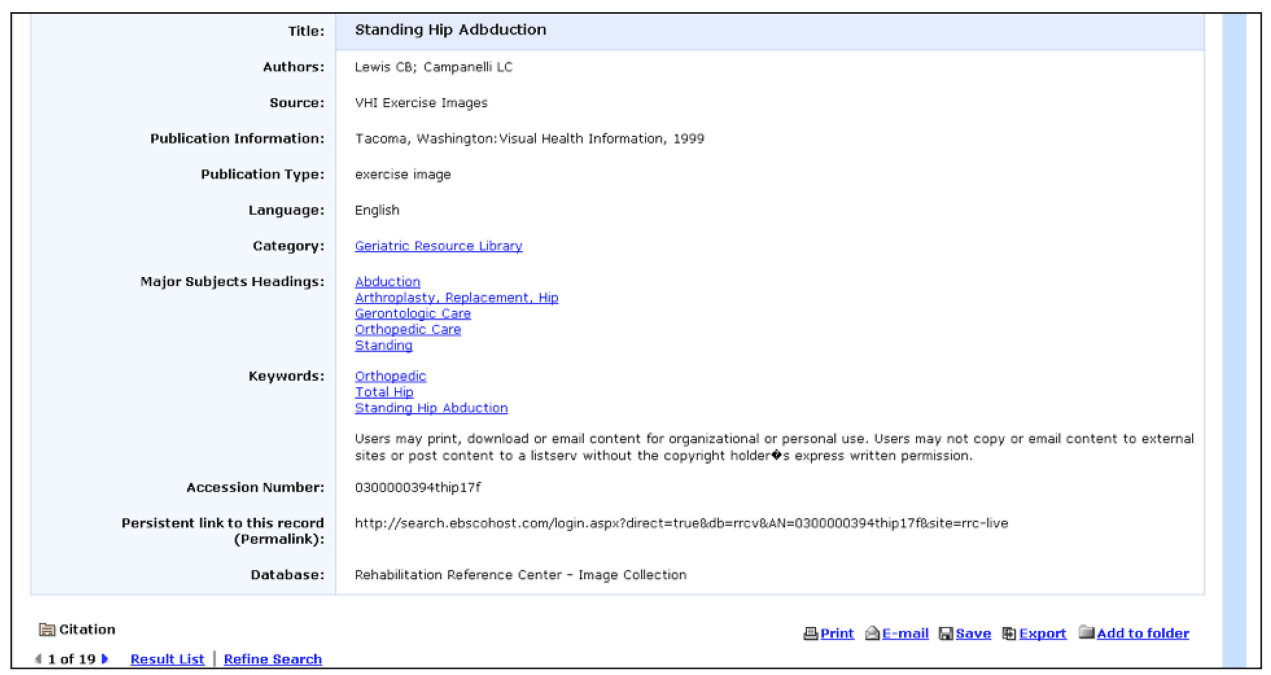

FIGURE 5 Subject heading and keywords assigned to an exercise image record pharmacokinetics, stability, actions, advice to patients, preparations, and references.

Patient Education RRC includes 2,600 evidence-based, medically illustrated patient handouts in English and Spanish. RRC's patient handouts are accredited by the Utilization Review Accreditation Committee (URAC). Customizeable print options allow the user to add personalized care notes. Figure 3 displays the custom printout options.

Exercise Images RRC has created 9,800 exercise images, using VCI, and indexed by CINAHL Rehabilitation Guide editorial team; custom printing allows users to add personalized care notes and order and sort exercises based on treatment plan. Over 40 categories of exercise images are available (such as Activities of Daily Living, Balance, Dynamic Exercise, Strength Training, Wheelchair, to name just a few). Within each category there may be various levels of subcategories. Each image is assigned subject headings and keywords. Figure 4 shows the exercise images retrieved for a search on hip replacement; Figure 5 shows the indexing and key words assigned to an exercise record for standing hip abduction.

Practice Resources A variety of resources are included in the database: Research Instruments (10 Attitude Measures, 71 Clinical Assessment Tools, 1 Neuropsychological Test, 7 Psychological tests, 24 Questionnaires, 11 Research Instruments, 68 Scales), 353 Practice Guidelines (clinical practice guidelines from National Guideline Clearinghouse), and 5 Featured Books (Orthopedic and Athletic Injury Evaluation Handbook; Therapeutic Exercise: Foundations and Techniques, Fifth edition; Modalities for Therapeutic Intervention, Fourth Edition; Developing Cultural Competence in Physical Therapy Practice; and Taber's Cyclopedic Medical Dictionary).
Health News This section offers news feeds from <http://www. healthday.com>.

\section{SEARCHING}

RRC contains a range of evidence-based clinical resources that can be retrieved in a variety of ways. The database has a Basic and Advanced Search, as well as an A-Z browse. The A-Z browse seems to be available for Diseases and Conditions, Drug Information, Patient Education, and Exercise Images. The Practice Resources browse function is subsumed under its various contents. The Advanced Search link reveals that the default search includes Clinical Reviews, Exercise Images, Drugs, Patient Education, Guidelines, Books, Research Instruments, and News. The interface is also set to look for related words and search within the full text. At any point the user can change the defaults under Advanced Search. The Basic Search helps somewhat with correct spelling if enough of the first few letters match a disease/ condition or drug in the database. Otherwise, there does not seem to

\section{Contact Information \\ Rehabilitation Reference Center EBSCO Publishing \\ 10 Estes St. \\ Ipswich, MA 01938 \\ Phone: (800) 653-2726 (U.S. and Canada) (978) 356-6500 (International) \\ Fax: (978) 356-6565 \\ E-mail: < information@ebscohost.com > \\ URL: <http://www.ebscohost.com/>}




\section{Rehabilitation Reference Center Review Scores Composite: $\star \star \star \star$}

The maximum number of stars in each category is 5 .

Content:

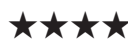

RRC gathers evidence-based information from a variety of reliable resources to create clinical reviews unique to RRC as well as exercise and patient handouts created and indexed by the CINAHL-RRC editorial team.

Searchability:

$\star \star \star \star$

RRC offers in-depth indexing, Basic, and Advanced searching, as well as lists to browse so the large variety of access points to a variety of materials is efficient to use at the point of care. Searchability would be improved by offering a spell-checker.

\section{Pricing Options:}

Pricing for RRC is determined on an individual basis and seems to be mostly based on the number of potential users.

\section{Contract Options:}

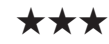

EBSCO's standard contract options apply.

be a spell-checker available. Figure 1 illustrates retrieval for a search on hip replacement sorted by All Results. The Administration module allows subscribing institutions to control which default is shown first, and the user can sort the results by clicking on any of the available tabs. Retrieved resources will depend upon the topic. The example given in Figure 1a shows the topic has retrievals for all resources. Refinements can also be made by subject headings, publication, and age as seen in Figure 1b. Figure 2 illustrates the extensive metaindexing and contents offered in a clinical review. Each clinical review offers a rated bibliography. Items in the bibliographies can be linked to an OpenURL resolver so users can easily link into further evidence-based articles depending upon the institution's subscription. Other EBSCO databases such as Nursing Reference Center and the CINAHLPlus database have similar Matrix codes for their evidence-based literature. Figure 3 highlights the custom print options RRC has available. $\mathrm{RRC}$ is, like the Nursing Reference Center, a point-of-care tool that gathers a variety of useful resources for rehabilitation therapists. It is easy to use in its browse function and does offer more sophisticated searching. The Clinical Reviews written for this database, the range of Exercise Images selected, classified, and indexed by RRC, and the patient handouts are especially helpful to its intended audience.

\section{Contract Provisions}

RRC uses the standard EBSCO license agreement. Resources are available on an unlimited use basis to authorized users of a given institution. Fair use regulations apply; RRC advises when additional written permission is needed from the copyright holder of the exercise images.

\section{Authentication}

EBSCO offers numerous options for authentication, including IP Address, Patterned IDs, Patron ID files, Referring URL, User ID and Password, Cookie Authentication, Athens authentication, Shibboleth authentication, and HTTPS authentication.

\section{Author's Selected References}

Boyd-Burnes, Mary Kate. "Ebsco's Nursing Reference Center." The Charleston Advisor 10, no.1 (2008): 34-37.

Dworak, Ellie. "New EBSCOhost Interface." The Charleston Advisor 10 , no.3 (2009): 55-58.

\section{About the Author}

Marcia Henry has been the Health Sciences Librarian and Physical Therapy, Kinesiology, and Communication Disorders Librarian at California State University Northridge since 1985. She was trained as a MEDLARS searcher in 1968, has interned at DIALOG in 1977, and has published many articles and one book mostly related to online searching.

Christina Mayberry is Science and Engineering Librarian at California State University, Northridge. She earned her M.L.I.S. at UCLA and has experience in academic medical libraries as a reference and information specialist and as a manager of a hospital library. 